\title{
Quantum electrodynamical shocks and solitons in astrophysical plasmas
}

\author{
M. Marklund, ${ }^{1}$ D. D. Tskhakaya, ${ }^{2,3}$ and P. K. Shukla ${ }^{1,4}$ \\ ${ }^{1}$ Department of Physics, Umeå University, SE-901 87 Umeå, Sweden \\ ${ }^{2}$ Institute of Physics, Georgian Academy of Sciences, 380077 Tbilisi, Georgia \\ ${ }^{3}$ Institute of Theoretical Physics, University of Innsbruck, A-6020 Innsbruck, Austria \\ ${ }^{4}$ Institut für Theoretische Physik IV, \\ Fakultät für Physik und Astronomie, \\ Ruhr-Universität Bochum, D-44780 Bochum, Germany
}

(Dated: September 23, 2021)

\begin{abstract}
The nonlinear propagation of low-frequency circularly polarized waves in a magnetized dusty plasma is analyzed. It is found that wave steepening and shock formation can take place due to the presence of nonlinear quantum vacuum effects, thus giving rise to ultra-intense electromagnetic shocks. Moreover, it is shown that solitary wave structures are admitted even under moderate astrophysical conditions. The results may have applications to astrophysical plasmas, as well as next generation laser interactions with laboratory plasmas containing dust clusters.

PACS numbers: 52.27.Fp, 52.35.Mw, 52.38.-r, 52.40.Db
\end{abstract}




\section{INTRODUCTION}

The extreme environment offered in many astrophysical situations, such as supernovae and binary mergers, in many cases give rise to new and exciting physics, e.g. neutrino-plasma interactions and gravitational waves. Furthermore, the next generation laser-plasma systems promises to take laboratory astrophysics into new realms, as tens to hundreds of petawatts, or even zetawatts, may be generated in femtosecond pulses [1]. Thus, as observational astronomy and cosmology grows even more accurate, and laboratory techniques continue to develop at the current rate, one will be able to probe new physics, such as neutron star equations of state [2] or the Unruh-Hawking effect [3].

Recently, it has been noted that the inclusion of quantum electrodynamics (QED) into plasma theory may give rise to novel wave modes [4, 5, 6], in particular in dusty plasmas 7]. These wave modes could propagate in a variety of environments, such as supernovae remnants, interstellar clouds, or laser-dusty plasma systems.

In Ref. 8] it was found, using the method of characteristics, that ultra-strong magnetic fields may induce shock front formation in electromagnetic radiation, analogous to hydrodynamic shocks. A possible application to such a scenario is magnetars, where field strengths of the order $10^{15} \mathrm{G}$ can be found.

In the present paper, we show that the nonlinear propagation of electromagnetic waves in a a weakly magnetized dusty plasma may give rise to wave steepening and shock formation. This effect is solely due to the novel QED effect of photon-photon scattering in a nonlinear quantum vacuum. The shock front formation may give rise to ponderomotive acceleration of charged particles in, e.g. interstellar clouds. The results are discussed for astrophysical energy scales.

\section{PULSE EVOLUTION}

In Ref. 7] the dispersion relation

$$
\frac{k^{2} c^{2}}{\omega^{2}} \approx \frac{4 \alpha}{45 \pi}\left[\left(\frac{E_{0}}{E_{S}}\right)^{2} \frac{k^{2} c^{2}}{\omega^{2}}+\left(\frac{c B_{0}}{E_{S}}\right)^{2}\right] \frac{k^{2} c^{2}}{\omega^{2}}-\frac{\omega_{p d}^{2} \gamma_{d}}{\omega_{c d}\left(\omega \gamma_{d}-\omega_{c d}\right)} .
$$

was found. The relation (11) is valid for low-frequency circularly electromagnetic polarized waves, with electric field $\mathbf{E}=E(\hat{\mathbf{x}}+i \hat{\mathbf{y}}) \exp (i k z-i \omega t)$, propagating along a magnetic 
field $\mathbf{B}_{0}=B_{0} \hat{\mathbf{z}}$ Moreover, the critical Schwinger field is given by $E_{S}=m_{0 e}^{2} c^{3} / e \hbar \sim 10^{18}$ $\mathrm{V} / \mathrm{m}$ (see, e.g. Ref. 9]), where $c$ is the speed of light in vacuum, $\hbar$ is Planck's constant divided by $2 \pi, m_{0 e}$ is the electron rest mass, $e$ is the absolute value of the electron charge, and $\omega_{c d}=q_{d} B_{0} / m_{0 d}$ and $\omega_{p d}=\left(n_{0 d} q_{d}^{2} / \epsilon_{0} m_{0 d}\right)^{1 / 2}$ is the dust gyro and plasma frequency, respectively. Here $n_{0 d}$ denotes the dust particle density in the laboratory frame, $q_{d}$ the dust charge, $m_{0 d}$ the dust particle rest mass, and $\alpha$ is the fine-structure constant.

Next, we introduce the dimensionless variables and parameters $\Omega=\omega / \omega_{c d}, K=k c / \omega_{p d}$, and $\kappa=(4 \alpha / 45 \pi)^{1 / 2} \omega_{p d} / \omega_{c d}$, and normalize the electric field according to $\mathcal{E}=E / E_{S}$. We consider the case of ultra-relativistic dust in a weak magnetic field, for which 7]

$$
K^{2} \Omega^{2}+\Omega^{3}=\kappa^{2}|\mathcal{E}|^{2} K^{4}
$$

holds.

The generic evolution equation for a slowly varying envelope $\mathcal{E}$ following from the dispersion relation (2) reads

$$
i \partial_{t}^{3} \mathcal{E}-\partial_{t}^{2} \partial_{z}^{2} \mathcal{E}+\kappa^{2}|\mathcal{E}|^{2} \partial_{z}^{4} \mathcal{E}=0
$$

where $i K \rightarrow \partial_{z}$ and $i \Omega \rightarrow-\partial_{t}$ has been used.

If the quantum parameter $\kappa \rightarrow 0$, from Eq. (2) we obtain the equation

$$
\left(i \partial_{t}-\partial_{z}^{2}\right) \mathcal{E}(t, z)=0
$$

yielding a linear traveling wave solution with the whistler-like dispersion relation $\Omega=-K^{2}$

In the case of small frequencies, $\Omega \ll K^{2}$, (the case with a dominant nonlinear term), we neglect the third order frequency $\Omega^{3}$ in Eq. (2) and obtain

$$
\partial_{t} \mathcal{E} \pm \kappa|\mathcal{E}| \partial_{z} \mathcal{E}=0
$$

Thus, letting $\mathcal{E}(t, z)=|\mathcal{E}(t, z)| \exp [i \varphi(t, z)]$ the amplitude $|\mathcal{E}(t, z)|$ satisfies the equation

$$
\partial_{t}|\mathcal{E}| \pm \kappa|\mathcal{E}| \partial_{z}|\mathcal{E}|=0
$$

while the phase is connected with the amplitude by the equation $\partial_{t} \varphi \pm \kappa|\mathcal{E}| \partial_{z} \varphi=0$. For simple waves [10] Eq. (6) yields the solution

$$
z= \pm \kappa|\mathcal{E}|+f(|\mathcal{E}|),
$$


i.e.

$$
|\mathcal{E}(t, z)|=f^{-1}(z \mp \kappa|\mathcal{E}| t)
$$

where the function $f^{-1}$ is the inverse function of $f$ and $f$ is determined by the initial condition at $t=0$. Thus, we have to define the initial condition $|\mathcal{E}(0, z)|=f(z)$ at $t=0$ and then find $z=f^{-1}(|\mathcal{E}|)$. As Eq. (7b ) shows, the wave steepening due to nonlinearity may occur. This can also be seen via Eq. (7a), from which we find that

$$
\partial_{z}|\mathcal{E}|=\frac{1}{ \pm \kappa t+f^{\prime}(|\mathcal{E}|)}
$$

where $f^{\prime}(x) \equiv \partial_{x} f(x)$.

(a) If $f^{\prime}(|\mathcal{E}|)<0$ (which can be interpreted as the initial function $|\mathcal{E}|=f(z)$ at $t=0$ decreasing with $z$ ), we have at time $t=-\left|f^{\prime}(|\mathcal{E}|)\right| / \kappa$ that $\partial_{z}|\mathcal{E}|=\infty$, if the plus is chosen in front of the $\kappa$-term in Eq. (8) .

(b) If we, on the other hand, have $f^{\prime}(|\mathcal{E}|)>0$, we choose the minus sign in front of the $\kappa$-term in Eq. (8), and obtain the shock front formation for a finite time.

Thus, we clearly have the shock wave formation at this instant [10]. Starting with a Gaussian pulse, case (a) implies the wave steepening and the shock front formation in the leading part of the pulse, while case (b) implies the wave steepening in the tail of the pulse, which can be seen by a simple spatial reflection symmetry. We note that in principle density gradient effects in the dusty plasma should be taken into account once significant steepening occurs, but this turns out to be a higher order effect.

In the weakly nonlinear case, we may use Eq. (44) to simplify the general pulse evolution equation (3), using $\partial_{z}^{2} \mathcal{E} \approx i \partial_{t} \mathcal{E}$. Assuming a slowly varying envelope $\mathcal{E}$, Eq. (3) yields

$$
i \partial_{t} \mathcal{E}-\partial_{z}^{2} \mathcal{E}-\kappa^{2}|\mathcal{E}|^{2} \mathcal{E}=0
$$

which is the well-known nonlinear Schrödinger equation with a focusing nonlinearity. Thus, such an equation admits the formation of a bright soliton [11] given by $\mathcal{E}(t, z)=$ $\mathcal{E}_{0} \operatorname{sech}\left(\mathcal{E}_{0} z\right) \exp \left(-i \mathcal{E}_{0}^{2} t / 2 \sqrt{2}\right)$, where $\mathscr{E}_{0}$ is the amplitude, and we have assumed that the soliton has the peak value at $t=0$ located at $z=0$. Thus, in the weakly nonlinear case, a balance between the dispersive and nonlinear effects may be struck. However, in practice, the coherence length of such structures could be short, due to e.g. plasma inhomogeneities. 
Moreover, while the one-dimensional bright soliton is stable, higher dimensional effects may yield instabilities. It is interesting thought that such structures could form with rather modest parameter values, as will be discussed next.

\section{DISCUSSION AND CONCLUSIONS}

The effect of the wave steepening and intense shock front formation presented here takes place whenever the electromagnetic intensity is high in the dusty plasma. Thus, a natural question arises: What are the situations when this may occur? As astrophysical systems offer extreme energy scales, these are natural places to look for QED effects, such as the one presented here. Moreover, the presence of dusty plasmas is well known in a wide range of astrophysical environments [12, 13, 14], such as supernovae [15, 16] and interstellar clouds 17]. In the case of Interstellar clouds, we have an expected dust density of the order $n_{0 d} \sim 10^{-1} \mathrm{~m}^{-3}$ [17], with a typical dust mass $m_{0 d} \sim 10^{6} m_{\text {proton }} \sim 10^{-21} \mathrm{~kg}$ [14]. A value $B_{0} \sim 10^{-8} \mathrm{G}$ (well within the limits for such environments) results in $\kappa \sim 10^{5}$. If the circularly polarized wave satisfy $E_{0} / E_{S} \approx 2 \times 10^{-5}$, a value appropriate at a distance of 1 AU from a supernova, we have $\kappa|\mathcal{E}| \sim 1$, thus giving an appreciable nonlinearity in Eqs. (6) and (9).

In Fig. 1 we have plotted the pulse evolution in two different cases. The upper panel represents the positive sign of Eq. (66), while the lower panel depicts the pulse evolution for the negative sign in Eq. (6). We have used the initial pulse amplitude $|\mathcal{E}(0, z)|=$ $\mathcal{E}_{0} \exp \left[-\left(z-z_{0}\right)^{2} / 2 a^{2}\right]$, with $z_{0}=3$ and $a=1$. Moreover, the initial amplitude satisfies $\kappa \mathcal{E}_{0}=0.7$, thus well within the limits posed by, e.g. interstellar clouds at $1 \mathrm{AU}$ from a supernova. We have plotted the evolution of $|\mathcal{E}(t, z)| / \mathcal{E}_{0}$. The effect of the nonlinearity can clearly be seen in the form of the wave steepening.

One interesting aspect of the shock front formation is the possibility of charged particle acceleration due to the ponderomotive force. However, before conclusions can be drawn on possible particle acceleration, analysis of mechanisms rendering the pulse wave front less steep must be made. 


\section{Acknowledgments}

This research was partially supported by the Swedish Research Council through the contract No. 621-2004-3217. 
[1] G.A. Mourou, T. Tajima, and S.V. Bulanov, Rev. Mod. Phys. 77, in press (2005).

[2] M. Shibata, Phys. Rev. Lett. 94, 201101 (2005).

[3] P. Chen and T. Tajima, Phys. Rev. Lett. 83, 256 (1999).

[4] M. Marklund, P.K. Shukla, L. Stenflo, G. Brodin, and M. Servin, Plasma Phys. Control. Fusion 47, in press (2005).

[5] M. Marklund, P.K. Shukla, G. Brodin, and L. Stenflo, Proceedings of the 12th International Congress on Plasma Physics, Nice (France) (25-29 October 2004). O3-0100; e-Proceedings available on line at http://hal.ccsd.cnrs.fr/ccsd-00003094/en (2004).

[6] L. Stenflo, G. Brodin, M. Marklund, and P.K. Shukla, J. Plasma Phys. 71, in press (2005).

[7] M. Marklund, L. Stenflo, P.K. Shukla, and G. Brodin, Phys. Plasmas 12, in press (2005).

[8] J.S. Heyl and L. Hernquist, Phys. Rev. D 58, 043005 (1998).

[9] J. Schwinger, Phys. Rev. 82, 664 (1951).

[10] L.D. Landau and E.M. Lifshitz, Fluids Mechanics (Pergamon Press, Oxford, 1959).

[11] A. Hasegawa, Plasma Instabilities and Nonlinear Effects (Springer-Verlag, Berlin, 1975).

[12] M. Horányi, T.W. Hartquist, O. Havnes, D.A. Mendis, and G.E. Morfill, Rev. Geophys. 42, RG4002 (2004).

[13] Y.K. Okamoto, H. Kataza, M. Honda, T. Yamashita, T. Onaka, J. Watanabe, T. Miyata, S. Sako, T. Fujiyoshi, and I. Sakon, Nature 431660 (2004).

[14] P.K. Shukla and A.A. Mamun, Introduction to Dusty Plasma Physics (Institute of Physics Publishing, Bristol, 2000).

[15] L. Dunne, S. Eales, R. Ivison, H. Morgan, and M. Edmunds, Nature 424285 (2003)

[16] O. Krause, S.M. Birkmann, G.H. Rieke, D. Lemke, U. Klaas, D.C. Hines, and K.D. Gordon, Nature 432596 (2004).

[17] D.A. Mendis and M. Rosenberg, Annu. Rev. Astron. Astrophys. 32419 (1994). 

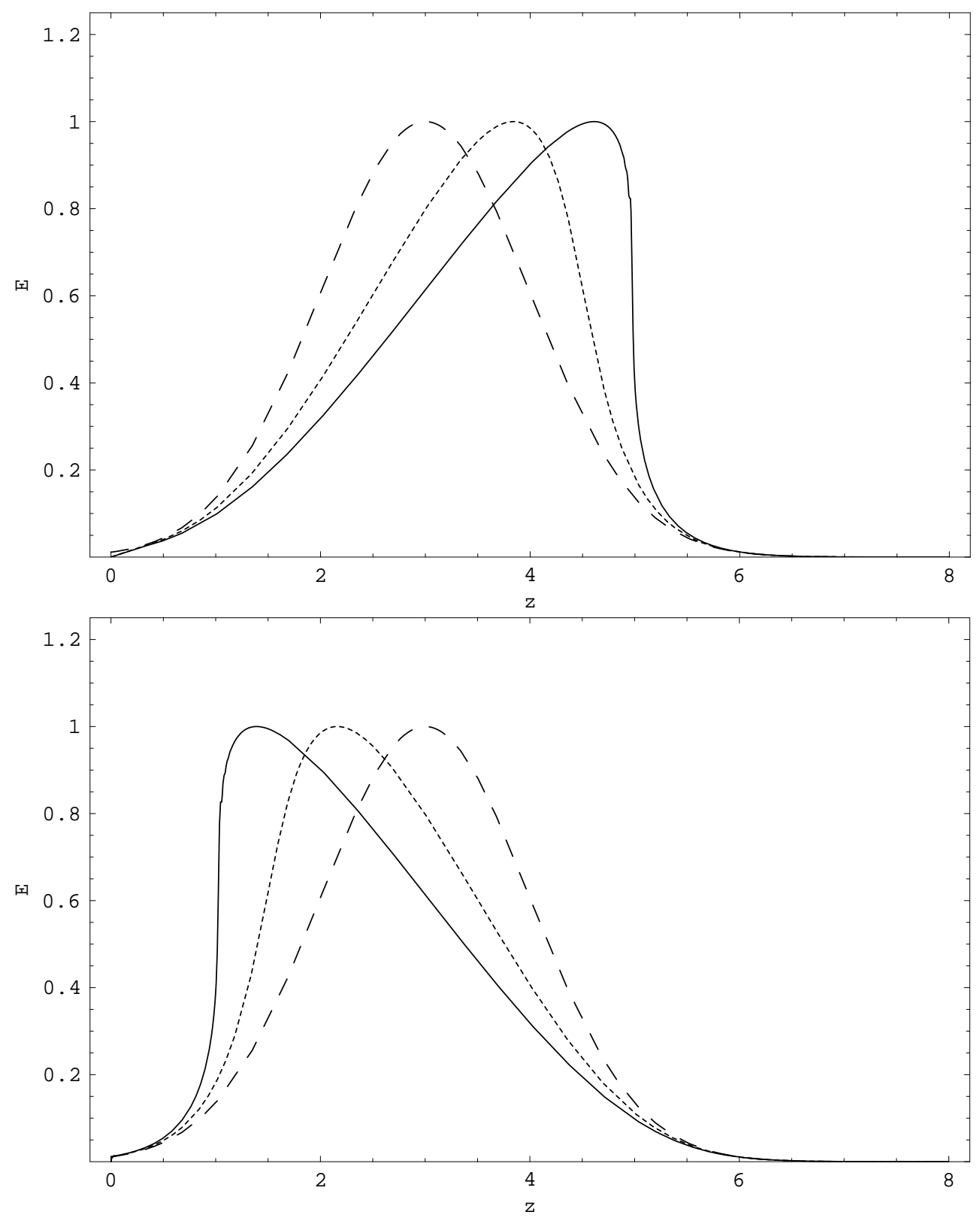

FIG. 1: The magnitude $|\mathcal{E}|$ of the normalized electric field relative to the initial value $\mathcal{E}_{0}$ plotted as a function of $z$ for three different times. At $t=0$ we have the dashed Gaussian curve, while at $t=12$ and $t=23$ we have the dotted and full curve, respectively. In the upper panel we have used the positive sign in Eq. (6). The wave steepening and shock front formation can clearly be seen. In the lower panel, we have instead used the negative sign in Eq. (6). As expected, the wave steepening and shock front formation now occurs at the trailing edge of the pulse. 